\title{
DOSAGE ÉLECTROPHOTOMÉTRIQUE DES VITAMINES A ET C DANS LE LAIT - INFLUENCE DE LA STABILISATION ET DE LA CONSERVATION
}

\author{
par
}

\author{
Mlle ANdrén VINET et M. PAUL MEUNIER
}

Il importe que les besoins en vitamines de l'enfant soient couverts, même si l'on doit recourir aux laits concentrés et conservés. Il est bon de noter que dans ces dernières années le dosage des vitamines $\mathrm{A}$ et $\mathrm{C}$ du lait est devenu très accessible aux chimistes et qu'il est aisé de suivre la conservation de ces facteurs au cours des opérations industrielles de traitement. Nous donnons ici les techniques qui nous ont donné satisfaction et quelques-uns de nos premiers résultats.

\section{$1^{\circ}$ Dosage de la vitamine A}

Après essai de différentes techniques pour la préparation de l'insaponifiable du lait, nous avons retenu la méthode de VAN Bever [1] sans isolement préalable des matières grasses.

\section{Extraction :}

$1^{0}$ Ajouter à $100 \mathrm{~cm}^{3}$ de lait, $100 \mathrm{~cm}^{3}$ d'alcool à $96^{\circ}$ et 20 grammes de potasse dans $10 \mathrm{~cm}^{3}$ d'eau. Porter le mélange au bain-marie à reflux pendant 2 heures;

$2^{\circ}$ Extraire à l'éther de pétrole par deux fois $100 \mathrm{~cm}^{3}$ et une fois $50 \mathrm{~cm}^{3}$;

$3^{\circ}$ Dessécher l'éther sur $\mathrm{SO}^{4} \mathrm{Na}^{2}$ pur anhydre. Evaporer après filtration et dessécher sous vide;

$4^{0}$ Reprendre le résidu sec dans $5 \mathrm{~cm}^{3}$ de chloroforme (1).

\section{Dosage :}

Pour le dosage proprement dit, nous avons suivi la technique de Meunier et Raoul [2].

Solutions nécessaires :

Réactif de CARr et Price (solution saturée de trichlorure d'antimoine dans le chloroforme : $30 \%$ environ de $\mathrm{SbCl}^{3}$ ).

Anhydride acétique pur.

Dans la euve soudée en verre Pyrex de l'électrophotomètre de l'un de nous [3], verser $0 \mathrm{~cm}^{3} 4$ de la solution chloroformique précédente. Ajouter 2 gouttes d'anhydride acétique et compléter au trait de jauge $\left(6 \mathrm{~cm}^{3}\right)$ par le réactif de Carr et Price.

(1) Le chloroforme doit être préalablement distillé et recueilli sur $\mathrm{CaCl}^{2}$ pour le priver de toute trace d'eau ou d'alcool qui précipiterait sur $\mathrm{SbCl}^{3}$. 
La solution se colore en bleu et, dans le cas de la vitamine A seulement, cette coloration s'affaiblit rapidement.

Faire les lectures à l'électrophotomètre de 15 en 15 secondes, jusqu'à 2 minutes environ, avec l'écran orangé de l'appareil.

Tracer la courbe cinétique des absorptions lumineuses et extrapoler jusqu'à l'origine. La différence entre ce chiffre et la valeur de l'absorption de base, multipliée par le coefficient d'étalonnage de l'appareil, donne le taux de vitamine A dans la prise d'essai. (L'absorption de base s'obtient en remplaçant le réactif de CARR et PRICE par le chloroforme et en faisant la lecture comme précédemment dans l'écran orangé).

En outre, nous avons déterminé parfois la teneur en carotène du lait (provitamine A) et cela par simple absorption dans l'écran bleu (même cuve) en admettant que c'est le seul pigment jaune de la fraction insaponifiable du lait. (Voir, par exemple, Deco [4].)

Voici les teneurs en vitamine $A$ que nous avons obtenues :

En U. I, par litre

\begin{tabular}{|c|c|}
\hline Lait normal $«$ Ofco $»$ (d'hiver) . . . . . . . . . . . . . & 1.010 \\
\hline Après stabilisation $(1$ jour $) \ldots \ldots \ldots \ldots \ldots$ & 885 \\
\hline Après stabilisation ( 4 jours) $\ldots \ldots \ldots \ldots \ldots \ldots$ & 890 \\
\hline Après stabilisation ( 5 jours) $\ldots \ldots \ldots \ldots \ldots \ldots$ & 720 \\
\hline Après stabilisation ( 3 semaines) $\ldots \ldots \ldots \ldots \ldots \ldots$ & 730 \\
\hline
\end{tabular}

Le carotène ne paraît pas se modifier sensiblement et oscille autour de 200 par litre (300 U. I.).

Ces laits ont été stabilisés de la façon suivante ; homogénéisation, dégazéification, préchauffage à $70^{\circ}$, stérilisation brève à $126^{\circ}$ en boîtes métalliques closes, refroidissement rapide. Comme l'a déjà fait remarquer M. JAviLLIER [5] et si l'on se reporte au tableau ci-dessus, "l'ensemble de ces opérations se traduit par une chute de l'activité vitaminique $\mathrm{A}$, chute qui n'est pas catastrophique et qui, une fois réalisée, ne s'intensifie guère avec le temps ».

Pour les laits condensés, nous avons obtenu les chiffres suivants :

En U. I. par litre

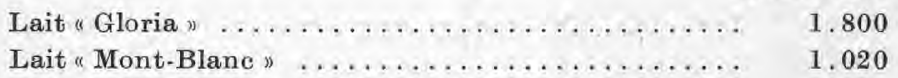

La perte de vitamine A est certainement plus sensible que pour les laits stabilisés, la concentration étant d'au moins moitié volume.

\section{$2^{\circ}$ Dosage de la vitamine C.}

Pour la détermination de la vitamine $\mathrm{C}$ antiscorbutique, nous avons employé la méthode de Meunier [6] basée sur l'étude cinétique de la décoloration du 2-6 dichlorophénol-indophénol. 
Cette vitamine étant éminemment oxydable, il est nécessaire de faire le dosage le plus rapidement possible. (Voir à ce sujet Meunier et Mentzer [6].)

\section{Extraction :}

Agiter pendant quelques instants $50 \mathrm{~cm}^{3}$ de lait et $50 \mathrm{~cm}^{3}$ de la solution aqueuse suivante :

$$
\begin{aligned}
& \mathrm{PO}^{3} \mathrm{H}-2 \% \\
& \mathrm{CCL}^{3} \mathrm{CO}^{2} \mathrm{H}-4 \% .
\end{aligned}
$$

Filtrer sur Buchner.

\section{Dosage :}

Ajouter à $5 \mathrm{~cm}^{3}$ de cette solution $5 \mathrm{~cm}^{3}$ d'une solution tampon à $p H 4$ (acide acétique normal $+1 / 5^{\mathrm{e}}$ de son volume d'une solution d'acétate de sodium à 136 grammes par litre).

Verser dans la cuve de l'électrophotomètre $5 \mathrm{~cm}^{3}$ de ce nouveau mélange, en prendre l'absorption de base (écran vert) et ajouter $1 \mathrm{~cm}^{3}$ de solution de dichlorophénol-indophénol(1). Mesurerl'absorption de 15 en 15 secondes pendant 2 minutes.

La valeur de la décoloration de l'indophénol à la $15^{\mathrm{e}}$ seconde multipliée par le coefficient d'étalonnage de l'appareil donne la quantité d'acide ascorbique contenue dans $2 \mathrm{~cm}^{3} 5$ de lait.

D’après les données que nous possédons, le lait de vache prélevé à la mamelle contient jusqu'à 25 milligrammes d'acide ascorbique par litre. Ce chiffre est d'ailleurs extrêmement variable et dépend en particulier de l'alimentation de la vache laitière. Nous n'avons jamais obtenu ce chiffre pour la raison que nous indiquions plus haut.

Voici quelques résultats obtenus sur le lait OFCO, avant et après stabilisation pratiquée comme il a déjà été indiqué à propos

\begin{tabular}{|c|c|c|}
\hline 4 & Avant stabilisation & Après stabilisation \\
\hline$\therefore$ & \multicolumn{2}{|c|}{ En milligrammes par litre } \\
\hline \multicolumn{3}{|l|}{ 1re Série: } \\
\hline Arrivée $\quad \ldots$ & 9,3 & 5,7 \\
\hline Après 3 jours & 1,8 & 5,6 \\
\hline Après 4 jours $\ldots . . . \ldots$ & 1,6 & 5,6 \\
\hline \multicolumn{3}{|l|}{$2^{\mathrm{e}}$ Série: } \\
\hline Arrivée $\ldots \ldots \ldots \ldots \ldots$ & 14,4 & 10 \\
\hline Après 4 heures . ....... & 9 & 10 \\
\hline Après 1 jour $\quad \ldots \ldots \ldots \ldots$ & 2,8 & 8,2 \\
\hline
\end{tabular}
de la vitamine A.

(1) Peser 5 mgr. de 2-6 dichlorophénol-indophénol dans une fiole jaugée de $50 \mathrm{~cm}^{3}$. Ajouter environ $40 \mathrm{~cm}^{3}$ d'eau bi-distillée récemment bouillie, $2 \mathrm{~cm}^{3}$ d'une solution. tampon à $p H$ 6,8 et complèter à $50 \mathrm{~cm}^{3}$ par l'eau bi-distillée. 
Comme il fallait s'y attendre, nous voyons que pour la vitamine C la chute d'activité au cours de la stabilisation atteint 30 à $40 \%$, mais, dans ce cas, les teneurs ne varient plus sensiblement ensuite.

\begin{tabular}{l|c|c}
\hline \hline \multicolumn{1}{c|}{ Laits condensés } & $\begin{array}{c}\text { Acide ascorbique } \\
\text { En milligrammes par litre }\end{array}$ & $\begin{array}{c}\text { Lactose } \\
\text { En grammes par litre }\end{array}$ \\
\hline Nestlé (non sueré) $\ldots \ldots \ldots \ldots \ldots$ & 30,24 & 83 \\
Gloria (non sucré) ........ & 11,8 & 68 \\
Mont-Blane (sucré) ....... & 34,5 & 95,6 \\
\hline \hline
\end{tabular}

\section{Conclusions}

Comme la pasteurisation dont les effets ont été déjà bien étudiés (voir un résumé récent dans KAy [8]), le procédé dit de "stabilisation ") altère peu l'activité en vitamine A du lait, mais abaisse nettement son titre en vitamine C (30 à $40 \%$ ).

Quant aux laits condensés, on remarquera que leurs teneurs en vitamine $\mathrm{A}$ et $\mathrm{C}$ diffèrent notablement d'un échantillon à l'autre.

En tous cas, et quel que soit le mode de conservation chcisi, il nous paraît utile de rechercher comment varient les teneurs en vitamines avec la provenance des laits et avec le temps.

Nous pensons qu'avec les méthodes chimiques relativement simples que nous possédons aujourd'hui, de tels contrôles peuvent rapidement entrer en pratique.

\section{BIBLIOGRAPHIE}

[1] Van Bever, Arch. Néerl. Physiol., 1938, 23, 552.

[2] P. Meunier et Y. Raoul. Bull. Soc. Chim. Biol., 1938.

[3] P. Meunier. Bull. Soc. Chim. Biol., 1937, 19, 113. Construit par Jовin et Yvon, Paris-Raspail.

[4] M. Deco. C. R. Soc. Biol., 1939, 130, 817, 819.

[5] M. Javiluter. C. R. Acad. Agr., 1940, 26, 369.

[6] P. Meunier. Bull. Soc. Chim. Biol., 1937, 19, 877.

[7] P. Meunier et Ch. Mentzer. C. R. Soc. Biol., 1938, 129, 1075.

[8] Kay. Nutri. Abs. Rev., 1939, 9, 1.

(Laboratoire de Chimie Biologique de la Faculté des Sciences de Paris, 96, Bd Raspail, Professeur M. Javillier). 